\title{
A Roadmap to the Brittle Bones of Cystic Fibrosis
}

\author{
Ashwini P. Gore, ${ }^{1}$ Soon Ho Kwon, ${ }^{1}$ and Antine E. Stenbit ${ }^{2}$ \\ ${ }^{1}$ Division of Endocrinology, Diabetes \& Medical Genetics, Department of Medicine, Medical University of South Carolina, \\ Charleston, SC 29425-6300, USA \\ ${ }^{2}$ Division of Pulmonary, Critical Care, Allergy \& Sleep Medicine, Department of Medicine, Medical University of South Carolina, \\ Charleston, SC 29425-6300, USA
}

Correspondence should be addressed to Antine E. Stenbit, stenbit@musc.edu

Received 1 September 2010; Accepted 5 November 2010

Academic Editor: Jan J. Štěpán

Copyright (C) 2011 Ashwini P. Gore et al. This is an open access article distributed under the Creative Commons Attribution License, which permits unrestricted use, distribution, and reproduction in any medium, provided the original work is properly cited.

Cystic fibrosis (CF) is an autosomal recessive disorder which despite advances in medical care continues to be a life-limiting and often fatal disease. With increase in life expectancy of the CF population, bone disease has emerged as a common complication. Unlike the osteoporosis seen in postmenopausal population, bone disease in CF begins at a young age and is associated with significant morbidity due to fractures, kyphosis, increased pain, and decreased lung function. The maintenance of bone health is essential for the CF population during their lives to prevent pain and fractures but also as they approach lung transplantation since severe bone disease can lead to exclusion from lung transplantation. Early recognition, prevention, and treatment are key to maintaining optimal bone health in CF patients and often require a multidisciplinary approach. This article will review the pathophysiology, current clinical practice guidelines, and potential future therapies for treating CF-related bone disease.

\section{Introduction}

Cystic fibrosis (CF) is an autosomal recessive disorder caused by defects in the cystic fibrosis transmembrane conductance regulator protein (CFTR), a chloride channel found in the epithelial tissues in the lungs, sinuses, pancreas, skin, and gastrointestinal tract. CF most commonly affects Caucasians and occurs with a frequency of 1 in 2000 to 3000 live births in the United States each year [1]. The defects in CFTR leads to alterations in the sodium, chloride, and water transport in the epithelial cells and in turn to changes in the viscosity and hydration of the fluids overlying the epithelial cells. The change in the fluid composition is partially responsible for several of the complications associated with the progression of CF such as chronic respiratory infections, pancreatic duct obstruction, pancreatic insufficiency, biliary obstruction, cirrhosis as well as distal intestinal obstruction syndrome. In addition to expression of CFTR in a variety of epithelial cells, its expression has been found in osteoblasts but its precise role in these cells remains to be elucidated $[2,3]$. The respiratory disease is hallmarked by bronchiectasis caused by cycles of infection, inflammation, and destruction of the airways. Airway clearance and aerosolized therapies have been a staple in the care of the CF patients for the past several decades and have lead to an improvement in the lifespan of the patients leading some to consider it now a life-limiting instead of a fatal disease. However, CF continues to lead to premature respiratory failure from repeated exacerbations, chronic infection by Pseudomonas aeruginosa, Staphylococcus aureus, Burkholderia cepacia, or other pathogenic organisms.

The rapid advancements in medical therapy and patient care discussed above have increased the median predicted survival age for patients with $\mathrm{CF}$ to 37.4 years (http://www.cff.org/). In the coming years, the number of patients with $\mathrm{CF}$ who are over the age of 18 will surpass those who are in the pediatric age group; currently approximately forty-six percent of the CF population in the United States is over 18 years of age. As a consequence, the various disease-related complications that were only seen in a subset of the CF population are now being seen in a larger number of patients, such as osteoporosis and osteopenia. The secondary bone loss seen in the CF population is multifactorial, however can cause significant morbidity in the adult population. 


\section{Bone Disease in CF}

Bone disease in patients with cystic fibrosis was first described over 3 decades ago and is characterized by decreased mineral density, increased fracture rates, and kyphosis $[4,5]$. Unlike bone loss seen in postmenopausal women, bone loss in the CF population begins at a young age and continues as the patient ages. The prevalence of bone disease in the CF population increases with age and has been correlated with severity of lung disease [6-11]. However, one is more likely to see an adult CF patient with low bone mineral density than with normal bone mineral density even with normal lung function.

A recent meta-analysis by Paccou et al. reported that the prevalence of osteoporosis and osteopenia in young adults with CF was $23.5 \%$ and $38 \%$, respectively [12]. Multiple cross-sectional studies have demonstrated an increased incidence of fractures in individuals with $\mathrm{CF}$ with vertebral fractures being the most common followed by rib fractures $[4,5,13-15]$. Another common skeletal problem seen in the CF population seen as early as the third decade of life is kyphosis $[13,16,17]$. Some studies have noted it to be between $10 \%-40 \%$ CF patients [16, 17]. The development of bone disease in the CF population can lead to significant issues with loss of lung function, deformities, and also increased pain issues. One can imagine that a vertebral or rib fracture would make it difficult to maintain a regimen of airway clearance that is needed for the prevention of $\mathrm{CF}$ exacerbations and the maintenance of lung function.

Although most of the pathologic consequences occur during adulthood in the CF population, there have been several studies that indicate that during childhood and puberty the CF patients achieve approximately half of the bone density than their healthy counterparts [18-22]. Puberty is especially important for the development of bone density and is a time where there is both peak growth velocity and bone density accrual. The reduction in the bone formation in the CF patients may be due to a combination of their delay in puberty, chronic infections, and hormonal imbalances. Numerous cross-sectional and longitudinal studies suggest that children and adolescents with cystic fibrosis fail to achieve adequate bone mass as compared to healthy controls during their pubertal growth spurt $[18-20,22]$. To compound this decrease in bone density in puberty and adolescence, clinical studies show annualized rates of loss in BMD in the CF patients approach those seen in postmenopausal women [23-25]. Lower baseline bone density coupled with increased losses puts $\mathrm{CF}$ individuals at higher risk of low BMD.

The maintenance of bone health is also essential for the CF population as they approach the time for lung transplantation. Severe bone disease can lead to exclusion from lung transplantation, which is often a life-saving treatment for many CF patients. The most effective strategies have been found to be early recognition, prevention, and treatment in this population. This article will discuss the pathogenesis, the current guidelines, and potential therapies for the CF population.

\section{Pathogenesis}

3.1. Histologically. Three of the studies that have looked at bone histomorphometric data in CF adults with low BMD have shown a decrease in cortical as well as trabecular bone volume and a decrease in connectivity [26-28]. At a cellular level, there is a disruption in the delicate balance between osteoblast and osteoclast activities. The osteoblastic activity is decreased in the bones of the CF patients due to decreased osteoblast number and their biosynthetic potential that is compounded by osteoclast activity which is increased, primarily through increased osteoclast number. The rise in bone resorption when compared to new bone formation leads to low bone density in this population even in patients who are clinically stable $[26,28]$. The exact cause of the disruption in this balance has not been completely been elucidated; however, there are studies to suggest that mutations in CFTR itself may play a role in a portion of the CF patients. Interestingly, even though vitamin D deficiency is very common in individuals with $\mathrm{CF}$ and is an important etiologic factor for the low BMD observed in this condition, osteomalacia is usually not a feature of CF-related bone disease [28].

\section{Possible Role of CFTR}

There are three observations that indicate that CFTR has a direct association with the loss in bone density in the CF patients. First, CFTR has been shown to be expressed in human osteoblasts, osteocytes, and osteoclasts [3]. Second, CF patients with at least one $\Delta \mathrm{F} 508$ allele had significantly lower Z-scores than those with other genotypes, which suggests a direct association between CF-related bone disease and the $\Delta$ F508 mutation [29]. Finally, experiments in CFTRdeficient mice demonstrated a decreased BMD with more cortical bone thinning and altered trabecular architecture as compared to control mice despite lacking other overt manifestations of CF (e.g., lung disease and pancreatic insufficiency) [30]. Together these findings suggest that loss of CFTR function may adversely affect bone density. However, the exact role of CFTR, specifically the $\Delta$ F508 allele in CF-related bone disease, is yet unclear.

In addition, the loss or reduction in CFTR activity has been shown to lead to chronic inflammation through a decrease in osteoprotegerin and a concomitant increase in prostaglandin E2 [2]. The alteration of osteoprotegerin and prostaglandin E2 increases the inflammation-driven bone resorption [2]. This would further indicate that there is a direct association between CFTR and the BMD in the CF patients. These studies have increased the interest in the possible role of CFTR gene in bone development and pathophysiological processes. Once again however, it is not known whether CFTR has a direct or indirect effect on bone formation.

\section{Factors Influencing Bone Loss}

The development of bone disease in the CF patients is thought also to be secondary to their chronic illness and is 
TABLe 1: Causes of decreased bone density in cystic fibrosis.

(i) Pancreatic insufficiency

(ii) Malnutrition and poor growth

(iii) Vitamin D, vitamin $\mathrm{K}$, and calcium insufficiency

(iv) CF-related diabetes

(v) Glucocorticoids

(vi) Sex steroid deficiency and delayed puberty

(vii) Chronic inflammation

(viii) Tobacco, alcohol, and caffeine use

(ix) Moderate to severe lung disease

(x) Lack of exercise, especially weight bearing

(xi) Organ transplant and immunosuppressive therapy

(xii) Medications (Depo-Provera, Megestrol Acetate, and Aluminium containing-antiacids)

(xiii) Possible role of CFTR.

influenced by multiple factors. It is essential to remember that nutrition, lung disease, and bone health are all related in this population. (Table 1).

\section{Malnutrition and Pancreatic Insufficiency}

Optimum nutrition is vital not only for pulmonary health but also for bone health [17,31-33]. Malnutrition seen in the adult CF patients is contributed by pancreatic insufficiency, increased catabolism due to chronic infections, and body images issues which afflicts many of the CF patients. A large portion of $\mathrm{CF}$ patients are pancreatic insufficient which leads to inadequate secretion of pancreatic enzymes and malabsorption of fat soluble vitamins (A, D, E, and $\mathrm{K})$, calcium, and other macronutrients which play a key role in bone formation [34]. Despite pancreatic enzyme replacement therapy, the majority of CF patients have body mass index that are in the low normal or malnutrition range. A great deal of energy is spent on dietary education and nutritional support in an attempt to improve the body mass index of the CF population. Low body mass index has been linked to low bone mineral density, especially in the adolescent and young adults $[35,36]$.

6.1. Vitamin Deficiencies. One vitamin deficiency which is prevalent in the CF population is Vitamin D deficiency, regardless of season or latitude [37-40]. In addition to pancreatic insufficiency and malabsorption described above, multiple other factors such as inactivity and decreased sunlight exposure, low body fat (reduced Vitamin D stores), reduced 25 hydroxylation, increased metabolic degradation, and decreased vitamin $\mathrm{D}$ binding protein have all been implicated as potential etiologies for the low vitamin D reserves.
In non-CF individuals, Vitamin D deficiency in children results in failure to achieve optimal height and peak bone mass or even overt rickets while in adults it is associated with impaired bone density due to decreased calcium absorption and hyperparathyroidism as well as osteomalacia. Although Vitamin D deficiency is commonly seen in CF population, osteomalacia in not a feature of CF-related bone disease, suggesting that other etiologies play an equally important role in pathogenesis [28].

Even with replacement therapy, vitamin $\mathrm{K}$ is known to be depleted in up to $40 \%$ of the CF patients. The significance of vitamin $\mathrm{K}$ to bone health has been linked to osteocalcin. A depletion of vitamin $\mathrm{K}$ has been associated with higher levels of undercarboxylated osteocalcin. Several studies have looked beneficial role of vitamin $\mathrm{K}$ supplementation specifically on the increased carboxylation of osteocalcin and improvement in bone formation [38, 41-44].

\section{Cystic Fibrosis-Related Diabetes}

Patients with CF can develop impaired glucose tolerance (IGT) or even frank diabetes due to the progressive pancreatic damage caused by defective acinar and ductular secretions. This type of pancreatic damage may not be similar to classic Type I or Type II diabetes; however, the resultant abnormalities in glucose and insulin regulation can affect bone health [45-47]. Just as in patients without CF, cystic fibrosis-related diabetes and IGT may play an important role in the development of bone loss [45].

\section{Gonadal Steroid Deficiency}

Puberty is a crucial period in which peak bone mass accrual occurs. CF individuals are frequently noted to have delayed puberty and decreased overall bone mineral accretion. Adolescents with CF have lower sex hormone levels as compared to healthy age matched controls, but most have normal values when adjusted for Tanner stage $[48,49]$. Hypogonadism can cause accelerated bone loss. The lower peak bone mass combined with increased bone losses may contribute to bone disease, but observational studies have not observed a consistent association $[48,49]$.

\section{Physical Inactivity}

Just as with some of the general population, the CF population is not as physically active as they should be. Although there are limitations with many of the patients with severe lung disease and there is social stigmata attached to the chronic cough that the CF patients have especially when exercising, any reduction in activity or weight bearing activity in this population has negative impact on their BMD. The CF patients are often burdened with a significant treatment burden and in the adult population frequent pulmonary or sinus exacerbation. For all of these reasons, there is a decrease in total activity hours and weight-bearing activities in this population even in light of the fact that it 
has been shown not only to improve bone health but also pulmonary health $[32,50-53]$.

\section{Recurrent Infections}

As stated previously, there is an association between the severity of lung disease and CF-related bone disease [5-10]. During acute exacerbations of lung disease, markers of bone turnover tend to rise and their levels drop after appropriate treatment with antibiotics, chest physical therapy, improved nutrition, and other supportive measures [54]. Systemic hormones, inflammatory cytokines, and localized growth factors can all affect bone remodeling [55]. Elevated levels of cytokines and growth factors like tumor necrosis factor$\alpha$ (TNF- $\alpha)$, vascular endothelial growth factor (VEGF) and IL-1, IL- 6 and IL-11 found in the serum and respiratory tract of CF patients may stimulate osteoclast-mediated bone resorption and inhibit bone formation [56].

\section{Glucocorticoids}

It is well established that glucocorticoids negatively impact bone health. Their primary effect on bone is to suppress formation of osteoblasts and promote apoptosis of osteoclasts and osteocytes. Systemic glucocorticoids can cause a rapid bone loss within the first few months of treatment, followed by a slower $2 \%-5 \%$ loss per year with chronic use [57]. CF patients with symptoms and signs of asthma or allergic bronchopulmonary aspergillosis are often treated with systemic or oral steroids to improve lung function. In most studies, the dose and duration of systemic steroid use impacted the decreased bone mineral density [58-62]. The use of inhaled corticosteroids, although associated with short term changes in markers of bone turnover, has not been shown to affect BMD or fracture risk, independent of severity of underlying lung disease $[58,62]$.

\section{Lung Transplantation and Immunosuppressive Therapy}

Lung transplantation may be potentially lifesaving in CF patients with end stage lung disease. Patients awaiting lung transplants almost always have a low BMD due to factors discussed above $[63,64]$. Immediately following the transplant, a rapid decline in BMD is noted and may be attributed to limited mobility, chronic steroid use, and use of other immunosuppressive agents [65-70]. This decreased BMD is associated with an increased fracture risk. Though BMD may stabilize or even increase a year or more after transplant, the fracture risk appears unchanged [65].

\section{Guidelines to Improve Bone Health in CF}

The clinical practice guidelines were outlined by the CF foundation committee for optimizing bone health in CF patients in The Journal of Clinical Endocrinology \& Metabolism in 2005 and are scheduled to be revised within the next year [13].
13.1. Screening for Bone Disease. The CF foundation bone health committee recommended that all adults with CF should have a baseline BMD at age 18 years. Children age 8 years or greater with other risk factors (i.e., ideal body weight $<90 \%$, FEV1 <50\% predicted, glucocorticoids $5 \mathrm{mg} /$ day or more for 90 days/year or longer, delayed puberty, or previous history of fractures) should also be screened.

The committee cautioned that while interpreting DEXA scan results, Z-scores should be used up to age 18 , Zor T-scores are nearly equivalent between ages 18 and 30, while T-scores should be used above the age of 30 . The recommendations are summarized in Table 2.

13.2. Preventive Strategies. The preservation of optimal bone health in CF requires a multisystemic approach. Good nutrition is critical in maintaining lean body mass and overall bone health. Vitamin and macronutrient deficiencies should be adequately repleted, and a BMI $>50 \%$ percentile should be targeted. Pulmonary infections should be treated aggressively, and every effort should be made to maintain adequate glycemic control and to minimize the use of steroids whenever possible. Weight bearing exercises are well known to increase BMD in healthy subjects and are highly recommended in preventing bone loss in CF individuals.

\section{Therapeutic Considerations}

14.1. Vitamin D Supplementation. As discussed previously, Vitamin D insufficiency is common among CF patients and is associated with bone disease. An optimum level has not been established but based on observations in non-CF patients that parathyroid hormone levels start to increase when $25 \mathrm{OH}$ Vit D levels fall below $30 \mathrm{ng} / \mathrm{mL}$, the CF foundation committee's consensus guidelines recommend a target $25 \mathrm{OH}$ Vit D level of $30 \mathrm{ng} / \mathrm{mL}$ or $75 \mathrm{nmol} / \mathrm{L}$. It should also be noted that the recommended daily allowance of Vit $\mathrm{D}$ for non-CF individuals will not be adequate to maintain target levels in CF patients. Various possible replacement strategies have been suggested, but the optimal Vitamin D analog and dose (D2, D3, calcifediol, calcitriol, and UV therapy) remains to be determined.

14.2. Calcium Supplementation. Calcium is an important component of bone infrastructure and is essential to the maintenance of skeletal health. Schulze et al. showed that increased calcium absorption in young women with CF was associated with increased rates of bone calcium deposition [34]. Since there are no long-term randomized controlled trials in CF patients, appropriate supplemental doses are not defined and national dietary reference intakes should be followed. The CF foundation bone health consensus committee recommends supplementation with $1300 \mathrm{mg}$ to $1500 \mathrm{mg}$ of elemental calcium daily.

14.3. Vitamin K Supplementation. Vitamin $\mathrm{K}$ is necessary for posttranslational activation of osteocalcin, which in turn is important for bone formation and mineralization [42, 43]. 
TABLE 2: Cystic fibrosis foundation guidelines for treatment of osteoporosis.

General Recommendations

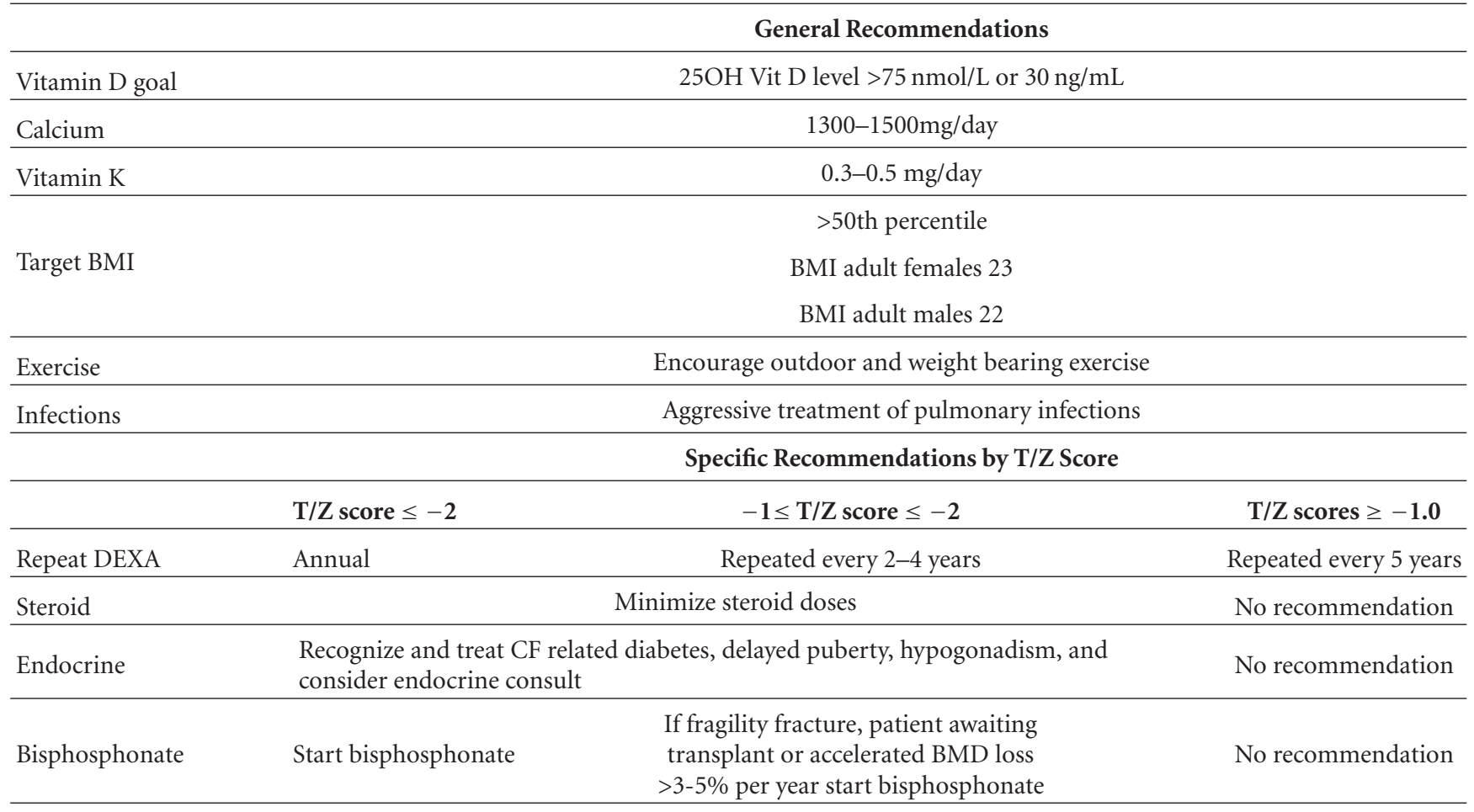

Clinical practice guidelines recommend supplementation with 0.3 to $0.5 \mathrm{mg}$ daily.

14.4. Gonadal Steroid Replacement Therapy. While adolescents with CF have lower sex hormone levels as compared to healthy age matched controls, most have normal values when adjusted for Tanner stage $[48,49]$. The risk/benefit ratio of sex steroid replacement has not been adequately studied. Gonadal hormone replacement is controversial and should be limited only to those patients who have persistently low levels $[13,71]$. Delayed puberty should be addressed by an experienced endocrinologist.

14.5. Antiresorptive Agents. Bisphosphonates are a class of drugs that chemically bind to calcium hydroxyl-apatite in bone and inhibit bone resorption through their inhibitory action on osteoclasts function and survival. Observational and randomized controlled trials (RCTs) on adults with CF have shown significant improvement in BMD with IV pamidronate and IV zoledronic acid as well as PO alendronate, but trials of these drugs in children with $\mathrm{CF}$ have not been performed [23-25, 65, 72, 73]. Oral agents are currently considered the first-line therapy for CF-related bone disease. The IV agents are frequently associated with side effects like fever, severe body aches, and bone pains $[25,65,72]$, but concomitant use of steroids, acetaminophen, and/or nonsteroidals may help reduce this pain and fever syndrome.

The current guidelines strongly recommend consideration of bisphosphonate therapy in all CF patients with $\mathrm{T} / \mathrm{Z}$-scores less than -2.0 and also in patients with $\mathrm{T} / \mathrm{Z}$ score between -1.0 and -2.0 , with a history of fragility fractures, those awaiting lung transplant, or who experience a BMD loss of $>3 \%-5 \%$ per year. It is essential that when a bisphosphonate is used that the Vitamin $\mathrm{D}$ and calcium are adequately replaced prior to and during therapy.

14.6. Recombinant Human Growth Hormone. Children with $\mathrm{CF}$ are noted to have poor linear growth and inadequate weight gain as well as low levels of IGF-1. The use of growth hormone in children with CF has been shown to have a beneficial effect on linear growth and weight gain. There is evidence to suggest that treatment with recombinant human growth hormone results in improvement in clinical status with decreased hospitalizations and courses of intravenous antibiotics, improvement in exercise tolerance, and bone accumulation [74-79]. Human recombinant growth hormone has not been studied as a treatment for low BMD in adults with cystic fibrosis $[74,75,77,78,80]$.

14.7. Teriparatide. Human parathyroid hormone has been shown to have both anabolic and catabolic effects on bone, depending on the dose and duration of use. While continuous exposure to high doses leads to bone resorption, daily low dose injections may actually increase osteoblast formation and bone growth [81-84]. Teriparatide (PTH 134 ) is the only anabolic agent available in the US for use in patients with advanced osteoporosis at high risk for fractures. Recombinant human (PTH 1-84) is available in Europe. It is contraindicated in children with open epiphyses but holds promise for CF adults with severe osteoporosis or prior history of fractures. There are currently no published studies 
of teriparatide in CF; however, this maybe a therapeutic option that should be considered and studied in the future for the adult population.

14.8. Newer Agents. Over the past few decades, researchers have made significant strides in the field of osteoporosis, which has led to an improved understanding of the regulation of bone remodeling. Exciting new treatment strategies have emerged and may potentially broaden our options for treatment of CF-related bone disease. Several of the therapies discussed are not clinically available currently but are under investigation and show promise to improve the bone health of the CF patients in the future.

14.8.1. Antiresorptive Agents. Denosumab, a human monoclonal antibody to RANKL (receptor activator of nuclear factor kappaB ligand), was recently approved by FDA for treatment of osteoporosis in postmenopausal women. Denosumab inhibits the maturation of osteoclasts by binding to RANKL [85]. The inhibition of osteoclast formation, function, and survival are responsible for decrease in osteoclast-mediated bone resorption. The improvement in lumbar and hip BMD seen with denosumab is at least comparable to bisphosphonates, if not superior. Denosumab also improves distal radius (cortical) bone density, an effect not seen with bisphosphonates [85]. Side effects noted with bisphosphonates such as delayed fracture healing, osteonecrosis of jaw, and femoral shaft fractures have not been observed in denosumab-treated individuals to date but further trials would be needed to assess the long-term effects of this medication [85]. The beneficial effect on both trabecular as well as cortical bone, combined with fewer side effects and the prospect of improved patient compliance due to twice-yearly dosing makes this an attractive consideration in CF-related bone disease.

Cathepsins are a family of proteases with collagen as their main target. Cathepsin K (CAT k) is unique to osteoclasts and plays a role in degradation of bone matrix proteins, including collagen. CATk inhibitors have an antiresorptive effect, with lesser inhibition of bone formation than bisphosphonates. CATk inhibitors have been shown in animal studies to increase cortical thickness and periosteal bone formation and are currently undergoing phase 3 trials. They may have the potential to improve long bone strength and prevent nonvertebral fractures $[86,87]$.

\subsubsection{Anabolic Agents}

PTH ligands and PTHrP. Antiresorptive agents can only increase bone mass but anabolic agents can also improve bone quality and strength, in addition to increasing bone mass. Currently, PTH [1-34] is the only approved anabolic agent in the US, and its duration of use is limited for 2 years. There is thus an unmet need for development of more anabolic agents.

Researchers have looked at various PTH ligands (PTH 1-31, PTH 1-28), which appear to have a more potent anabolic effect than the hormone itself (PTH 1-34) [88, 89].
Parathyroid hormone-related protein ( $\mathrm{PTHrP})$ is a hormone produced by mature osteoblasts and is structurally similar to PTH. Unlike PTH, which has both anabolic and catabolic effects on bone, PTHrP appears to be purely anabolic when administered intermittently. Studies in animals have shown an increase in trabecular bone volume, osteoblast number, bone mineralization rates, biomechanical strength, and BMD [90]. Human studies show increase in BMD similar to that with PTH, but without significant hypercalcemia or other adverse effects as observed with PTH. There is no activation of bone resorption at therapeutically effective doses [91-93].

14.8.3. Calcilytic Agents. Allosteric modulations of the Gprotein coupled calcium-sensing receptor in the parathyroid gland can affect PTH release. Positive allosteric modulators decrease PTH production, while negative modulators increase it. Calcilytics or calcium sensing channel antagonists are negative modulators, which when administered orally, can cause a pulsatile increase in PTH production. Animal studies look promising; unfortunately, a human trial of ronacaleret was discontinued due to a poor effect on BMD. Another agent is currently in phase 2 trials [94].

\subsubsection{Modulation of the Wnt Signaling Pathway}

Antisclerostin Antibody. A large family of extracellular glycoproteins, called Wnt proteins, are key regulators of bone remodeling and other cellular processes [95-97]. Sclerosteosis, an autosomal recessive disorder characterized by increased bone mass, mainly in the skull and in long bones, results from a mutation in the SOST gene, which codes for sclerostin [98]. A deficiency of sclerostin results in increased Wnt signaling and high bone mass. Transgenic mice that overexpress sclerostin are seen to have low bone mass and increased susceptibility to fractures [99] while mice deficient in sclerostin show increased bone density [100]. Treatment with sclerostin antibody has been associated with an increase in bone mass and strength in various animal models [101103]. In a phase I trial in 72 healthy men and postmenopausal women, a single subcutaneous dose of sclerostin antibody was associated with a statistically significant increase in the levels of bone formation markers propeptide of type I procollagen (P1NP), osteocalcin and bone-specific alkaline phosphatase (BSAP), a dose-dependent decrease in bone resorption marker C-telopeptide, and a 5.3\% increase in lumbar spine BMD [104]. Antisclerostin antibody seems promising as a potentially effective anabolic agent for the treatment of low bone mass in individuals with CF.

Anti-Dkk1 Antibody. Dickoppf1 (DKK1) is a naturally occurring Wnt pathway antagonist which works by preventing the interaction between two key Wnt pathway coreceptors, LRP5/6 and the frizzled Wnt pathway receptor, which results in inhibition of Wnt signal transduction and impaired bone formation [105]. DKK1 inhibition increases trabecular bone volume and bone formation in rats [106, 107]. Antibodies to dickkopf-1 could be used as an anabolic agent for the treatment of patients with low bone mass. 
Activin Inhibitors. Activin is a member of the bone morphogenic protein (BMP)/transforming growth factor (TGF) $\beta$ superfamily of polypeptides and stimulates the release of FSH by the pituitary gland [108]. In bone, activin binds to activin receptor IIA, increases osteoclastogenesis, and is a negative regulator of bone mass. Its effects on bone formation are more complex and unclear [109].

ACE-011, a protein formed by fusing soluble activin receptor type II to IgG-Fc, was shown to decreases bone resorption in ovariectomized mice and enhance bone formation in intact animals [110]. Data in cynomolgus monkeys shows a significantly higher BMD and trabecular bone volume after injection of ACE-011 [111]. In phase I trials, the administration of a single dose to 48 postmenopausal women resulted in an increase in levels of bone-specific alkaline phosphatase and a decrease in C-telopeptides [112]. Activin inhibitors hold promise as new anabolic therapy for patients with low bone mass and increased susceptibility for fractures.

\section{Conclusion}

CF-related bone disease is a common complication in the aging CF population. It is multifactorial in origin, affects young individuals, and is associated with significant morbidity. Despite increasing research in the field, numerous questions about pathogenesis and appropriate therapy still remain. A multisystemic, multidisciplinary approach is required to tackle bone disease in the CF population. Various therapies are currently available for treatment of postmenopausal osteoporosis but these have not been studied in individuals with CF. Clinical trial data is especially limited in the pediatric age group. The new Cystic Fibrosis Foundation guidelines for the treatment of bone disease are expected to be published in the next year. Exciting new therapies are currently under development, and investigation and hold promise for reducing the burden of secondary bone disease in individuals with $\mathrm{CF}$.

\section{References}

[1] R. C. Stern, “The diagnosis of cystic fibrosis," New England Journal of Medicine, vol. 336, no. 7, pp. 487-491, 1997.

[2] L. Le Heron, C. Guillaume, F. Velard et al., "Cystic fibrosis transmembrane conductance regulator (CFTR) regulates the production of osteoprotegerin (OPG) and prostaglandin (PG) E in human bone," Journal of Cystic Fibrosis, vol. 9, no. 1, pp. 69-72, 2010.

[3] E. F. Shead, C. S. Haworth, A. M. Condliffe, D. J. McKeon, M. A. Scott, and J. E. Compston, "Cystic fibrosis transmembrane conductance regulator (CFTR) is expressed in human bone," Thorax, vol. 62, no. 7, pp. 650-651, 2007.

[4] R. M. Aris, J. B. Renner, A. D. Winders et al., "Increased rate of fractures and severe kyphosis: sequelae of living into adulthood with cystic fibrosis," Annals of Internal Medicine, vol. 128, no. 3, pp. 186-193, 1998.

[5] S. L. Elkin, A. Fairney, S. Burnett et al., "Vertebral deformities and low bone mineral density in adults with cystic fibrosis: a cross-sectional study," Osteoporosis International, vol. 12, no. 5, pp. 366-372, 2001.
[6] L. K. Bachrach, C. W. Loutit, R. B. Moss, and R. Marcus, "Osteopenia in adults with cystic fibrosis," American Journal of Medicine, vol. 96, no. 1, pp. 27-34, 1994.

[7] D. S. Hardin, R. Arumugam, D. K. Seilheimer, A. LeBlanc, and K. J. Ellis, "Normal bone mineral density in cystic fibrosis," Archives of Disease in Childhood, vol. 84, no. 4, pp. 363-368, 2001.

[8] J. P. Lambert, "Osteoporosis: a new challenge in cystic fibrosis," Pharmacotherapy, vol. 20, no. 1, pp. 34-51, 2000.

[9] E. M. Laursen, C. Mølgaard, K. F. Michaelsen, C. Koch, and J. Müller, "Bone mineral status in 134 patients with cystic fibrosis," Archives of Disease in Childhood, vol. 81, no. 3, pp. 235-240, 1999.

[10] A. S. Neri, I. Lori, G. Taccetti et al., "Alteration of bone mineral density in cystic fibrosis adults," Chest, vol. 130, no. 6, pp. 1952-1953, 2006.

[11] J. Robertson and K. MacDonald, "Prevalence of bone loss in a population with cystic fibrosis," British Journal of Nursing, vol. 19, no. 10, pp. 636-639, 2010.

[12] J. Paccou, N. Zeboulon, C. Combescure, L. Gossec, and B. Cortet, "The prevalence of osteoporosis, osteopenia, and fractures among adults with cystic fibrosis: a systematic literature review with meta-analysis," Calcified Tissue International, vol. 86, no. 1, pp. 1-7, 2010.

[13] R. M. Aris, P. A. Merkel, L. K. Bachrach et al., "Consensus statement: guide to bone health and disease in cystic fibrosis," Journal of Clinical Endocrinology and Metabolism, vol. 90, no. 3, pp. 1888-1896, 2005.

[14] S. P. Conway, B. Oldroyd, K. G. Brownlee, S. P. Wolfe, and J. G. Truscott, "A cross-sectional study of bone mineral density in children and adolescents attending a Cystic Fibrosis Centre," Journal of Cystic Fibrosis, vol. 7, no. 6, pp. 469-476, 2008.

[15] I. Sermet-Gaudelus, M. Castanet, G. Retsch-Bogart, and R. M. Aris, "Update on cystic fibrosis-related bone disease: a special focus on children," Paediatric Respiratory Reviews, vol. 10, no. 3, pp. 134-142, 2009.

[16] R. C. Henderson and B. B. Specter, "Kyphosis and fractures in children and young adults with cystic fibrosis," Journal of Pediatrics, vol. 125, no. 2, pp. 208-212, 1994.

[17] R. B. Parasa and N. Maffulli, "Musculoskeletal involvement in cystic fibrosis," Bulletin: Hospital for Joint Diseases, vol. 58, no. 1, pp. 37-44, 1999.

[18] G. S. Bhudhikanok, M. C. Wang, R. Marcus, A. Harkins, R. B. Moss, and L. K. Bachrach, "Bone acquisition and loss in children and adults with cystic fibrosis: a longitudinal study," Journal of Pediatrics, vol. 133, no. 1, pp. 18-27, 1998.

[19] M. L. Bianchi, G. Romano, S. Saraifoger, D. Costantini, C. Limonta, and C. Colombo, "BMD and body composition in children and young patients affected by cystic fibrosis," Journal of Bone and Mineral Research, vol. 21, no. 3, pp. 388396, 2006.

[20] H. M. Buntain, R. M. Greer, P. J. Schluter et al., "Bone mineral density in Australian children, adolescents and adults with cystic fibrosis: a controlled cross sectional study," Thorax, vol. 59, no. 2, pp. 149-155, 2004.

[21] E. Gronowitz, M. Garemo, A. Lindblad, D. Mellström, and B. Strandvik, "Decreased bone mineral density in normalgrowing patients with cystic fibrosis," Acta Paediatrica, vol. 92, no. 6, pp. 688-693, 2003.

[22] R. C. Henderson and C. D. Madsen, "Bone density in children and adolescents with cystic fibrosis," Journal of Pediatrics, vol. 128, no. 1, pp. 28-34, 1996. 
[23] R. M. Aris, G. E. Lester, M. Caminiti et al., "Efficacy of alendronate in adults with cystic fibrosis with low bone density," American Journal of Respiratory and Critical Care Medicine, vol. 169, no. 1, pp. 77-82, 2004.

[24] I. Chapman, H. Greville, P. R. Ebeling et al., "Intravenous zoledronate improves bone density in adults with cystic fibrosis (CF)," Clinical Endocrinology, vol. 70, no. 6, pp. 838846, 2009.

[25] A. Papaioannou, C. C. Kennedy, A. Freitag et al., "Alendronate once weekly for the prevention and treatment of bone loss in Canadian adult cystic fibrosis patients (CFOS Trial)," Chest, vol. 134, no. 4, pp. 794-800, 2008.

[26] S. L. Elkin, S. Vedi, S. Bord, N. J. Garrahan, M. E. Hodson, and J. E. Compston, "Histomorphometric analysis of bone biopsies from the iliac crest of adults with cystic fibrosis," American Journal of Respiratory and Critical Care Medicine, vol. 166, no. 11, pp. 1470-1474, 2002.

[27] C. S. Haworth, A. J. Freemont, A. K. Webb et al., "Hip fracture and bone histomorphometry in a young adult with cystic fibrosis," European Respiratory Journal, vol. 14, no. 2, pp. 478-479, 1999.

[28] C. S. Haworth, A. K. Webb, J. J. Egan et al., "Bone histomorphometry in adult patients with cystic fibrosis," Chest, vol. 118, no. 2, pp. 434-439, 2000.

[29] S. J. King, D. J. Topliss, T. Kotsimbos et al., "Reduced bone density in cystic fibrosis: $\Delta$ F508 mutation is an independent risk factor," European Respiratory Journal, vol. 25, no. 1, pp. 54-61, 2005.

[30] C. K. Haston, W. Li, A. Li, M. Lafleur, and J. E. Henderson, "Persistent osteopenia in adult cystic fibrosis transmembrane conductance regulator-deficient mice," American Journal of Respiratory and Critical Care Medicine, vol. 177, no. 3, pp. 309-315, 2008.

[31] M. P. Boyle, "Update on maintaining bone health in cystic fibrosis," Current Opinion in Pulmonary Medicine, vol. 12, no. 6, pp. 453-458, 2006.

[32] D. D. Frangolias, P. D. Paré, D. L. Kendler et al., "Role of exercise and nutrition status on bone mineral density in cystic fibrosis," Journal of Cystic Fibrosis, vol. 2, no. 4, pp. 163170, 2003.

[33] R. C. Henderson and C. D. Madsen, "Bone mineral content and body composition in children and young adults with cystic fibrosis," Pediatric Pulmonology, vol. 27, no. 2, pp. 8084, 1999.

[34] K. J. Schulze, K. O. O’Brien, E. L. Germain-Lee, S. L. Booth, A. Leonard, and B. J. Rosenstein, "Calcium kinetics are altered in clinically stable girls with cystic fibrosis," Journal of Clinical Endocrinology and Metabolism, vol. 89, no. 7, pp. 3385-3391, 2004.

[35] A. B. Grey, R. W. Ames, R. D. Matthews, and I. R. Reid, “Bone mineral density and body composition in adult patients with cystic fibrosis," Thorax, vol. 48, no. 6, pp. 589-593, 1993.

[36] O. Tschopp, A. Boehler, R. Speich et al., "Osteoporosis before lung transplantation: association with low body mass index, but not with underlying disease," American Journal of Transplantation, vol. 2, no. 2, pp. 167-172, 2002.

[37] D. S. Donovan, A. Papadopoulos, R. B. Staron et al., "Bone mass and vitamin D deficiency in adults with advanced cystic fibrosis lung disease," American Journal of Respiratory and Critical Care Medicine, vol. 157, no. 6, part 1, pp. 1892-1899, 1998.

[38] V. Grey, S. Atkinson, D. Drury et al., "Prevalence of low bone mass and deficiencies of vitamins $\mathrm{D}$ and $\mathrm{K}$ in pediatric patients with cystic fibrosis from 3 Canadian centers," Pediatrics, vol. 122, no. 5, pp. 1014-1020, 2008.

[39] A. B. Haaber, A. M. Rosenfalck, B. Hansen, J. Hilsted, and S. Larsen, "Bone mineral metabolism, bone mineral density, and body composition in patients with chronic pancreatitis and pancreatic exocrine insufficiency," International Journal of Pancreatology, vol. 27, no. 1, pp. 21-27, 2000.

[40] W. B. Hall, A. A. Sparks, and R. M. Aris, "Vitamin $\mathrm{d}$ deficiency in cystic fibrosis," International Journal of Endocrinology, vol. 2010, Article ID 218691, 9 pages, 2010.

[41] R. M. Aris, D. A. Ontjes, S. A. Brown, W. Chalermskulrat, I. Neuringer, and G. E. Lester, "Carboxylated osteocalcin levels in cystic fibrosis," American Journal of Respiratory and Critical Care Medicine, vol. 168, no. 9, p. 1129, 2003.

[42] P. Nicolaidou, I. Stavrinadis, I. Loukou et al., "The effect of vitamin $\mathrm{K}$ supplementation on biochemical markers of bone formation in children and adolescents with cystic fibrosis," European Journal of Pediatrics, vol. 165, no. 8, pp. 540-545, 2006.

[43] D. Drury, V. L. Grey, G. Ferland, C. Gundberg, and L. C. Lands, "Efficacy of high dose phylloquinone in correcting vitamin K deficiency in cystic fibrosis," Journal of Cystic Fibrosis, vol. 7, no. 5, pp. 457-459, 2008.

[44] M. S. Fewtrell, C. Benden, J. E. Williams et al., "Undercarboxylated osteocalcin and bone mass in 8-12 year old children with cystic fibrosis," Journal of Cystic Fibrosis, vol. 7, no. 4, pp. 307-312, 2008.

[45] D. S. Harbin, A. Leblanc, G. Marshall, and D. K. Seilheimer, "Mechanisms of insulin resistance in cystic fibrosis," American Journal of Physiology, vol. 281, no. 5, pp. E1022-E1028, 2001.

[46] K. Soejima and B. H. Landing, "Osteoporosis in juvenileonset diabetes mellitus: morphometric and comparative studies," Pediatric Pathology, vol. 6, no. 2-3, pp. 289-299, 1986.

[47] P. S. Zeitler, S. Travers, and M. S. Kappy, "Advances in the recognition and treatment of endocrine complications in children with chronic illness," Advances in pediatrics, vol. 46, pp. 101-149, 1999.

[48] S. R. Boas, D. A. Cleary, P. A. Lee, and D. M. Orenstein, "Salivary testosterone levels in male adolescents with cystic fibrosis," Pediatrics, vol. 97, no. 3, pp. 361-363, 1996.

[49] M. Johannesson, B. M. Landgren, G. Csemiczky, L. Hjelte, and C. Gottlieb, "Female patients with cystic fibrosis suffer from reproductive endocrinological disorders despite good clinical status," Human Reproduction, vol. 13, no. 8, pp. 20922097, 1998.

[50] K. Hind, J. G. Truscott, and S. P. Conway, "Exercise during childhood and adolescence: a prophylaxis against cystic fibrosis-related low bone mineral density?. Exercise for bone health in children with cystic fibrosis," Journal of Cystic Fibrosis, vol. 7, no. 4, pp. 270-276, 2008.

[51] T. E. Kottke, C. J. Caspersen, and C. S. Hill, "Exercise in the management and rehabilitation of selected chronic diseases," Preventive Medicine, vol. 13, no. 1, pp. 47-65, 1984.

[52] K. J. MacKelvie, K. M. Khan, M. A. Petit, P. A. Janssen, and H. A. McKay, "A school-based exercise intervention elicits substantial bone health benefits: a 2-year randomized controlled trial in girls," Pediatrics, vol. 112, no. 6, part 1, p. e447, 2003.

[53] M. Sahlberg, B. Strandvik, and A. Barak, "Trampolines are useful in the treatment of cystic fibrosis patients," Pediatric Pulmonology, vol. 40, no. 5, pp. 464-465, 2005. 
[54] R. M. Aris, A. R. Stephens, D. A. Ontjes et al., "Adverse alterations in bone metabolism are associated with lung infection in adults with cystic fibrosis," American Journal of Respiratory and Critical Care Medicine, vol. 162, no. 5, pp. 1674-1678, 2000.

[55] S. C. Manolagas and R. L. Jilka, "Mechanisms of disease: bone marrow, cytokines, and bone remodeling-emerging insights into the pathophysiology of osteoporosis," New England Journal of Medicine, vol. 332, no. 5, pp. 305-311, 1995.

[56] J. Jacquot, O. Tabary, and A. Clement, "Hyperinflammation in airways of cystic fibrosis patients: what's new?" Expert Review of Molecular Diagnostics, vol. 8, no. 4, pp. 359-363, 2008.

[57] S. C. Manolagas and R. S. Weinstein, "New developments in the pathogenesis and treatment of steroid-induced osteoporosis," Journal of Bone and Mineral Research, vol. 14, no. 7, pp. 1061-1066, 1999.

[58] R. B. Hubbard, C. J. P. Smith, L. Smeeth, T. W. Harrison, and A. E. Tattersfield, "Inhaled corticosteroids and hip fracture: a population-based case-control study," American Journal of Respiratory and Critical Care Medicine, vol. 166, no. 12, part 1, pp. 1563-1566, 2002.

[59] D. Yen and D. Hedden, "Multiple vertebral compression fractures in a patient treated with corticosteroids for cystic fibrosis," Canadian Journal of Surgery, vol. 45, no. 5, pp. 383384, 2002.

[60] S. P. Conway, A. M. Morton, B. Oldroyd et al., "Osteoporosis and osteopenia in adults and adolescents with cystic fibrosis: prevalence and associated factors," Thorax, vol. 55, no. 9, pp. 798-804, 2000.

[61] J. M. Borro, A. Solé, M. De La Torre, A. Pastor, and V. Tarazona, "Steroid withdrawal in lung transplant recipients," Transplantation Proceedings, vol. 37, no. 9, pp. 3991-3993, 2005.

[62] T. P. Van Staa, H. G. M. Leufkens, and C. Cooper, "Use of inhaled corticosteroids and risk of fractures," Journal of Bone and Mineral Research, vol. 16, no. 3, pp. 581-588, 2001.

[63] E. Shane, S. J. Silverberg, D. Donovan et al., "Osteoporosis in lung transplantation candidates with end-stage pulmonary disease," American Journal of Medicine, vol. 101, no. 3, pp. 262-269, 1996.

[64] S. Teramoto, T. Matsuse, Y. Ouchi, and E. Shane, "Osteoporosis in lung transplantation candidates with end stage pulmonary disease," American Journal of Medicine, vol. 103, no. 4, pp. 334-335, 1997.

[65] R. M. Aris, G. E. Lester, J. B. Renner et al., "Efficacy of pamidronate for osteoporosis in patients with cystic fibrosis following lung transplantation," American Journal of Respiratory and Critical Care Medicine, vol. 162, no. 3, part 1, pp. 941-946, 2000.

[66] R. M. Aris, I. P. Neuringer, M. A. Weiner, T. M. Egan, and D. Ontjes, "Severe osteoporosis before and after lung transplantation," Chest, vol. 109, no. 5, pp. 1176-1183, 1996.

[67] P. N. Chhajed, M. Tamm, M. A. Malouf, and A. R. Glanville, "Lung transplantation: management and complications," The Indian Journal of Chest Diseases \& Allied Sciences, vol. 44, no. 1, pp. 31-43, 2002.

[68] A. Dosanjh, "A review of nutritional problems and the cystic fibrosis lung transplant patient," Pediatric Transplantation, vol. 6, no. 5, pp. 388-391, 2002.

[69] I. P. Neuringer, P. Noone, R. K. Cicale, K. Davis, and R. M. Aris, "Managing complications following lung transplanta- tion," Expert Review of Respiratory Medicine, vol. 3, no. 4, pp. 403-423, 2009.

[70] S. Quattrucci, M. Rolla, G. Cimino et al., "Lung transplantation for cystic fibrosis: 6-year follow-up," Journal of Cystic Fibrosis, vol. 4, no. 2, pp. 107-114, 2005.

[71] C. Landon and R. G. Rosenfeld, "Short stature and pubertal delay in male adolescents with cystic fibrosis. Androgen treatment," American Journal of Diseases of Children, vol. 138, no. 4, pp. 388-391, 1984.

[72] C. S. Haworth, P. L. Selby, A. K. Webb, E. B. Mawer, J. E. Adams, and T. J. Freemont, "Severe bone pain after intravenous pamidronate in adult patients with cystic fibrosis," Lancet, vol. 352, no. 9142, pp. 1753-1754, 1998.

[73] I. Pande and D. J. Hosking, "Oral antiresorptive therapy," Current Rheumatology Reports, vol. 7, no. 1, pp. 71-77, 2005.

[74] D. S. Hardin, C. Ahn, C. Prestidge, D. K. Seilheimer, and K. J. Ellis, "Growth hormone improves bone mineral content in children with cystic fibrosis," Journal of Pediatric Endocrinology and Metabolism, vol. 18, no. 6, pp. 589-595, 2005.

[75] D. S. Hardin, J. Rice, M. E. Doyle, and A. Pavia, "Growth hormone improves protein catabolism and growth in prepubertal children with HIV infection," Clinical Endocrinology, vol. 63, no. 3, pp. 259-262, 2005.

[76] M. Vanderwel and D. S. Hardin, "Growth hormone normalizes pubertal onset in children with cystic fibrosis," Journal of Pediatric Endocrinology and Metabolism, vol. 19, no. 3, pp. 237-244, 2006.

[77] D. S. Hardin, J. Rice, C. Ahn et al., "Growth hormone treatment enhances nutrition and growth in children with cystic fibrosis receiving enteral nutrition," Journal of Pediatrics, vol. 146, no. 3, pp. 324-328, 2005.

[78] A. Schibler, R. Von Der Heiden, P. Birrer, and P. E. Mullis, "Prospective randomised treatment with recombinant human growth hormone in cystic fibrosis," Archives of Disease in Childhood, vol. 88, no. 12, pp. 1078-1081, 2003.

[79] D. S. Hardin, T. Ferkol, C. Ahn et al., "A retrospective study of growth hormone use in adolescents with cystic fibrosis," Clinical Endocrinology, vol. 62, no. 5, pp. 560-566, 2005.

[80] D. S. Hardin, B. Adams-Huet, D. Brown et al., "Growth hormone treatment improves growth and clinical status in prepubertal children with cystic fibrosis: results of a multicenter randomized controlled trial," Journal of Clinical Endocrinology and Metabolism, vol. 91, no. 12, pp. 49254929, 2006.

[81] D. W. Dempster, F. Cosman, M. Parisien, V. Shen, and R. Lindsay, "Anabolic actions of parathyroid hormone on bone," Endocrine Reviews, vol. 14, no. 6, pp. 690-709, 1993.

[82] J. S. Finkelstein, A. Hayes, J. L. Hunzelman, J. J. Wyland, H. Lee, and R. M. Neer, "The effects of parathyroid hormone, alendronate, or both in men with osteoporosis," New England Journal of Medicine, vol. 349, no. 13, pp. 1216-1226, 2003.

[83] R. L. Jilka, "Molecular and cellular mechanisms of the anabolic effect of intermittent PTH," Bone, vol. 40, no. 6, pp. 1434-1446, 2007.

[84] R. M. Neer, C. D. Arnaud, J. R. Zanchetta et al., "Effect of parathyroid hormone (1-34) on fractures and bone mineral density in postmenopausal women with osteoporosis," New England Journal of Medicine, vol. 344, no. 19, pp. 1434-1441, 2001.

[85] S. R. Cummings, J. S. Martin, M. R. McClung et al., "Denosumab for prevention of fractures in postmenopausal women with osteoporosis," New England Journal of Medicine, vol. 361, no. 8, pp. 756-765, 2009. 
[86] H. G. Bone, M. R. McClung, C. Roux et al., "Odanacatib, a cathepsin-K inhibitor for osteoporosis: a two-year study in postmenopausal women with low bone density," Journal of Bone and Mineral Research, vol. 25, no. 5, pp. 937-947, 2010.

[87] J. A. Eisman et al., "Odanacatib in the treatment of postmenopausal women with low bone mineral density: threeyear continued therapy and resolution of effect," Journal of Bone and Mineral Research. In press.

[88] M. J. Horwitz, M. B. Tedesco, S. M. Sereika, B. W. Hollis, A. Garcia-Ocaña, and A. F. Stewart, "Direct comparison of sustained infusion of human parathyroid hormonerelated protein-(1-36) [hPTHrP-(1-36)] versus hPTH-(1$34)$ on serum calcium, plasma 1,25-dihydroxyvitamin D concentrations, and fractional calcium excretion in healthy human volunteers," Journal of Clinical Endocrinology and Metabolism, vol. 88, no. 4, pp. 1603-1609, 2003.

[89] M. J. Horwitz, M. B. Tedesco, S. M. Sereika et al., "Continuous PTH and PTHrP infusion causes suppression of bone formation and discordant effects on 1,25(OH) vitamin D," Journal of Bone and Mineral Research, vol. 20, no. 10, pp. 1792-1803, 2005.

[90] A. F. Stewart, R. L. Cain, D. B. Burr, D. Jacob, C. H. Turner, and J. M. Hock, "Six-month daily administration of parathyroid hormone and parathyroid hormone-related protein peptides to adult ovariectomized rats markedly enhances bone mass and biomechanical properties: a comparison of human parathyroid hormone 1-34, parathyroid hormonerelated protein 1-36, and SDZ- parathyroid hormone 893," Journal of Bone and Mineral Research, vol. 15, no. 8, pp. 15171525, 2000.

[91] M. J. Horwitz, M. B. Tedesco, C. Gundberg, A. GarciaOcana, and A. F. Stewart, "Short-term, high-dose parathyroid hormone-related protein as a skeletal anabolic agent for the treatment of postmenopausal osteoporosis," Journal of Clinical Endocrinology and Metabolism, vol. 88, no. 2, pp. 569-575, 2003.

[92] M. J. Horwitz, M. B. Tedesco, S. M. Sereika et al., "Safety and tolerability of subcutaneous $\operatorname{PTHrP}(1-36)$ in healthy human volunteers: a dose escalation study," Osteoporosis International, vol. 17, no. 2, pp. 225-230, 2006.

[93] H. Plotkin, C. Gundberg, M. Mitnick, and A. F. Stewart, "Dissociation of bone formation from resorption during 2week treatment with human parathyroid hormone-related peptide-(1-36) in humans: potential as an anabolic therapy for osteoporosis," Journal of Clinical Endocrinology and Metabolism, vol. 83, no. 8, pp. 2786-2791, 1998.

[94] E. M. Brown, "The calcium-sensing receptor: physiology, pathophysiology and CaR-based therapeutics," Sub-cellular Biochemistry, vol. 45, pp. 139-167, 2007.

[95] R. Baron and G. Rawadi, "Minireview: targeting the Wnt/ $\beta$ catenin pathway to regulate bone formation in the adult skeleton," Endocrinology, vol. 148, no. 6, pp. 2635-2643, 2007.

[96] D. A. Glass and G. Karsenty, "Minireview: in vivo analysis of Wnt signaling in bone," Endocrinology, vol. 148, no. 6, pp. 2630-2634, 2007.

[97] V. Krishnan, H. U. Bryant, and O. A. MacDougald, "Regulation of bone mass by Wnt signaling," Journal of Clinical Investigation, vol. 116, no. 5, pp. 1202-1209, 2006.

[98] J. C. Gardner, R. L. Van Bezooijen, B. Mervis et al., "Bone mineral density in sclerosteosis; affected individuals and gene carriers," Journal of Clinical Endocrinology and Metabolism, vol. 90, no. 12, pp. 6392-6395, 2005.
[99] D. G. Winkler, M. K. Sutherland, J. C. Geoghegan et al., "Osteocyte control of bone formation via sclerostin, a novel BMP antagonist," EMBO Journal, vol. 22, no. 23, pp. 62676276, 2003.

[100] X. Li, M. S. Ominsky, Q. T. Niu et al., "Targeted deletion of the sclerostin gene in mice results in increased bone formation and bone strength," Journal of Bone and Mineral Research, vol. 23, no. 6, pp. 860-869, 2008.

[101] X. Li, M. S. Ominsky, K. S. Warmington et al., "Sclerostin antibody treatment increases bone formation, bone mass, and bone strength in a rat model of postmenopausal osteoporosis," Journal of Bone and Mineral Research, vol. 24, no. 4, pp. 578-588, 2009.

[102] M. S. Ominsky, X. Li, F. J. Asuncion et al., "RANKL inhibition with osteoprotegerin increases bone strength by improving cortical and trabecular bone architecture in ovariectomized rats," Journal of Bone and Mineral Research, vol. 23, no. 5, pp. 672-682, 2008.

[103] M. S. Ominsky, F. Vlasseros, J. Jolette et al., "Two doses of sclerostin antibody in cynomolgus monkeys increases bone formation, bone mineral density, and bone strength," Journal of Bone and Mineral Research, vol. 25, no. 5, pp. 948-959, 2010.

[104] D. Padhi et al., "Single-dose, placebo-controlled, randomized study of AMG 785, a sclerostin monoclonal antibody," Journal of Bone and Mineral Research. In press.

[105] M. V. Semënov, X. Zhang, and XI. He, "DKK1 antagonizes Wnt signaling without promotion of LRP6 internalization and degradation," Journal of Biological Chemistry, vol. 283, no. 31, pp. 21427-21432, 2008.

[106] J. Li, I. Sarosi, R. C. Cattley et al., "Dkk1-mediated inhibition of Wnt signaling in bone results in osteopenia," Bone, vol. 39, no. 4, pp. 754-766, 2006.

[107] F. Morvan, K. Boulukos, P. Clément-Lacroix et al., "Deletion of a single allele of the Dkk1 gene leads to an increase in bone formation and bone mass," Journal of Bone and Mineral Research, vol. 21, no. 6, pp. 934-945, 2006.

[108] E. Canalis, A. N. Economides, and E. Gazzerro, "Bone morphogenetic proteins, their antagonists, and the skeleton," Endocrine Reviews, vol. 24, no. 2, pp. 218-235, 2003.

[109] M. Centrella, T. L. McCarthy, and E. Canalis, "ActivinA binding and biochemical effects in osteoblast-enriched cultures from fetal-rat parietal bone," Molecular and Cellular Biology, vol. 11, no. 1, pp. 250-258, 1991.

[110] R. S. Pearsall, E. Canalis, M. Cornwall-Brady et al., "A soluble activin Type IIA receptor induces bone formation and improves skeletal integrity," Proceedings of the National Academy of Sciences of the United States of America, vol. 105, no. 19, pp. 7082-7087, 2008.

[111] R. J. Fajardo, R. K. Manoharan, R. S. Pearsall et al., "Treatment with a soluble receptor for activin improves bone mass and structure in the axial and appendicular skeleton of female cynomolgus macaques (Macaca fascicularis)," Bone, vol. 46, no. 1, pp. 64-71, 2010.

[112] C. Deal, "Potential new drug targets for osteoporosis," Nature Clinical Practice Rheumatology, vol. 5, no. 1, pp. 20-27, 2009. 


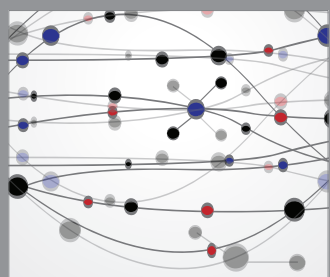

The Scientific World Journal
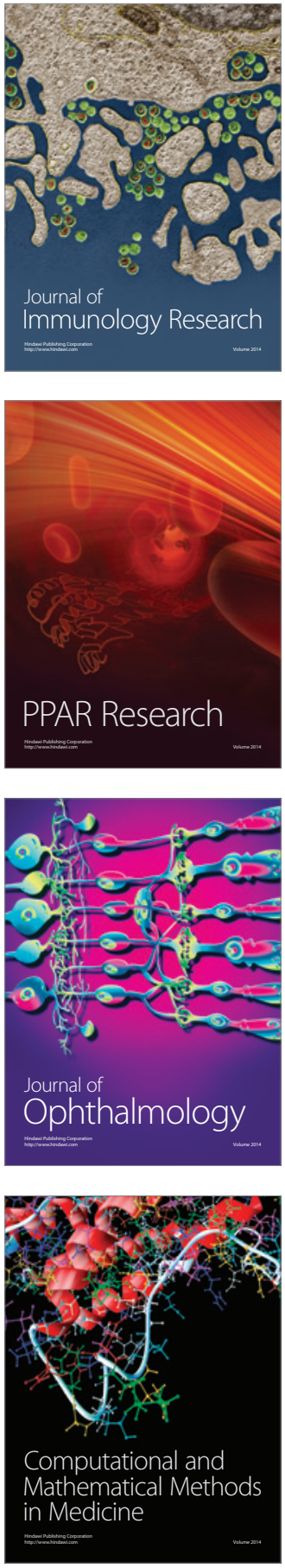

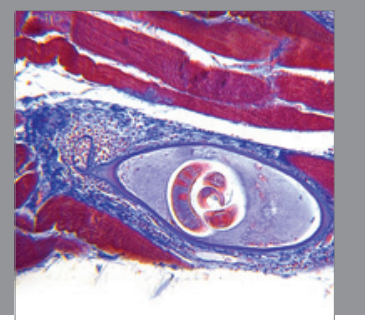

Gastroenterology

Research and Practice
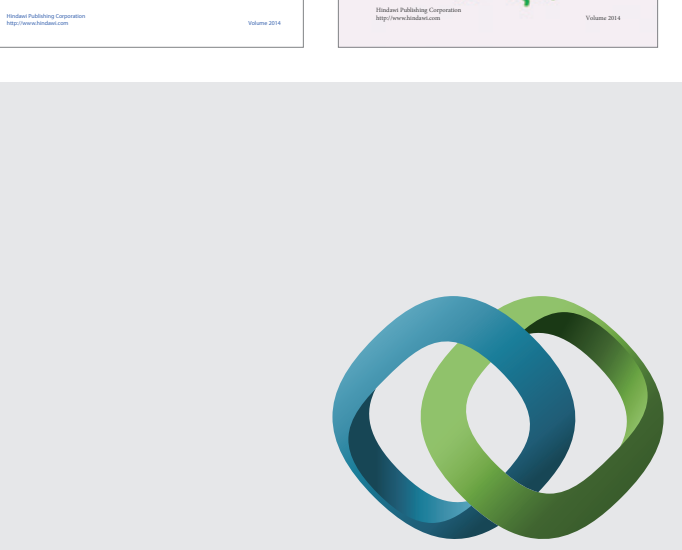

\section{Hindawi}

Submit your manuscripts at

http://www.hindawi.com
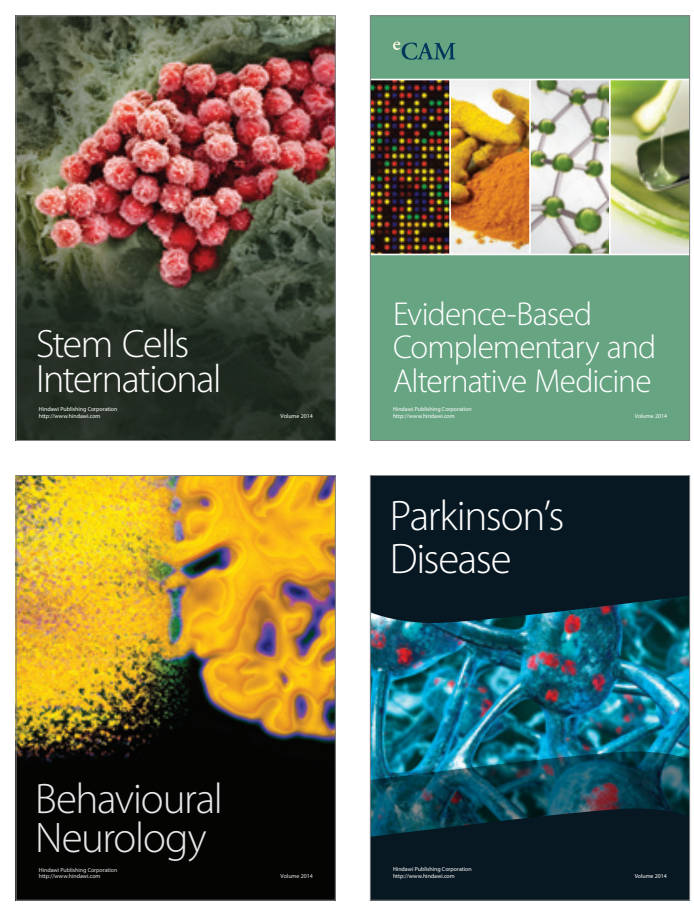

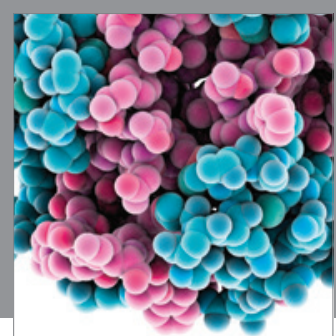

Journal of
Diabetes Research

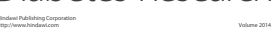

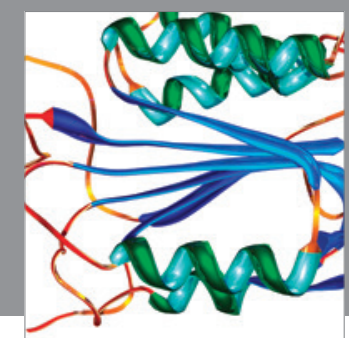

Disease Markers
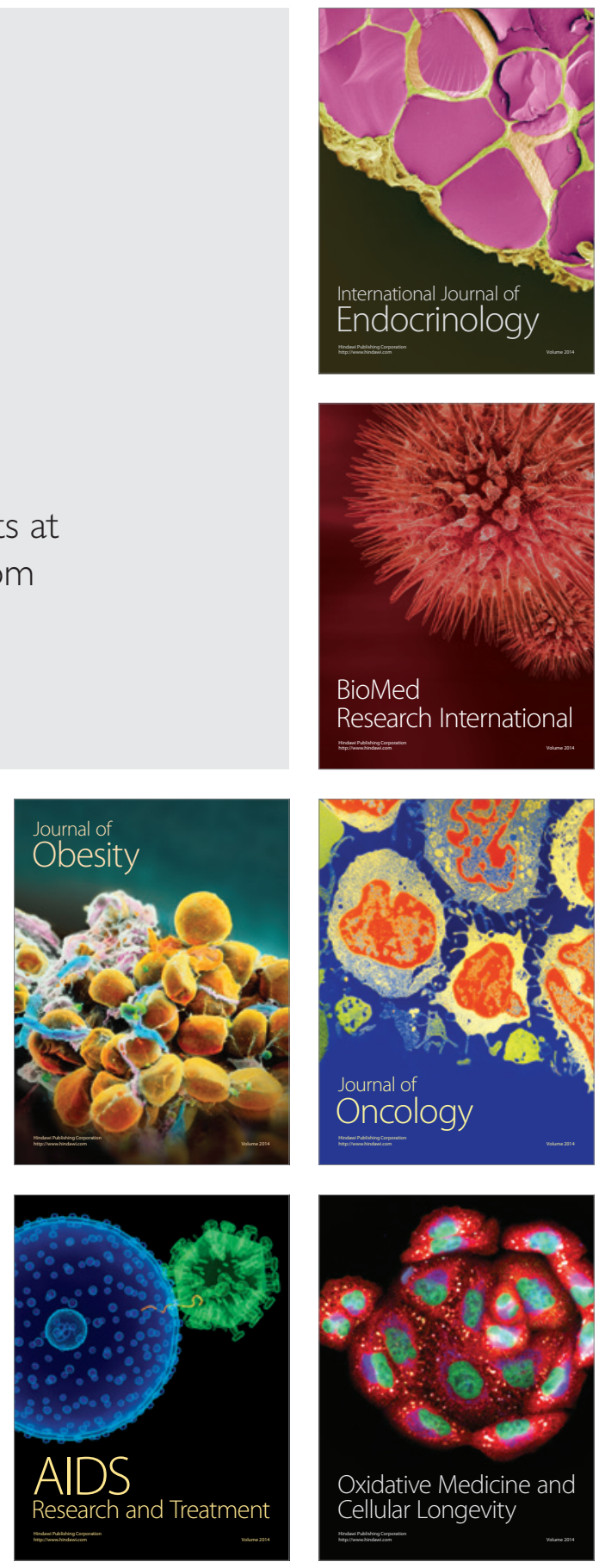